\title{
Three-Month Feeding Integration With Bifidobacterium Strains Prevents Gastrointestinal Symptoms in Healthy Newborns
}

\author{
Irene Aloisio ${ }^{1 \dagger}$, Flavia Prodam ${ }^{2,3 \dagger}$, Enza Giglione ${ }^{2}$, Nicole Bozzi Cionci ${ }^{1}$, Arianna Solito ${ }^{2}$, \\ Simonetta Bellone ${ }^{2,3}$, Loredana Baffoni ${ }^{1}$, Luca Mogna ${ }^{4}$, Marco Pane ${ }^{4}$, Gianni Bona ${ }^{2}$ and \\ Diana Di Gioia ${ }^{1 *}$ \\ ${ }^{1}$ Department of Agricultural and Food Sciences, University of Bologna, Bologna, Italy, ${ }^{2}$ Department of Health Sciences, \\ Università degli Studi del Piemonte Orientale, Novara, Italy, ${ }^{3}$ Interdisciplinary Research Center of Autoimmune Diseases \\ (IRCAD), Università degli Studi del Piemonte Orientale, Novara, Italy, ${ }^{4}$ Biolab Reserach, Novara, Italy
}

OPEN ACCESS

Edited by:

Nadiya V. Boyko,

Uzhhorod National University, Ukraine

Reviewed by:

Brian D. Piccolo,

Arkansas Children's Nutrition Center,

United States

Irena Rogelj;

Biotechnical Faculty, University of

Ljubljana, Slovenia

*Correspondence:

Diana Di Gioia

diana.digioia@unibo.it

tThese authors have contributed equally to this work.

Specialty section: This article was submitted to Food Microbiology,

a section of the journal

Frontiers in Nutrition

Received: 30 November 2017 Accepted: 23 April 2018

Published: 25 May 2018

Citation:

Aloisio I, Prodam F, Giglione E, Bozzi

Cionci N, Solito A, Bellone $S$,

Baffoni L, Mogna L, Pane M, Bona G and Di Gioia D (2018) Three-Month

Feeding Integration With

Bifidobacterium Strains Prevents

Gastrointestinal Symptoms in Healthy

Newborns. Front. Nutr. 5:39

doi: 10.3389/fnut.2018.00039
Infantile functional gastrointestinal disorders are common in the first months of life. Their pathogenesis remains unknown although evidences suggest multiple independent causes, including gut microbiota modifications. Feeding type, influencing the composition of intestinal microbiota, could play a significant role in the pathogenesis. Previous studies supported probiotic supplementation success against colics, however mainly Lactobacillus spp. were tested. The aim of this study was to evaluate the effectiveness against functional gastrointestinal disorders of a Bifidobacterium breve based probiotic formulation including in the study both breast-fed and bottle-fed subjects. Two hundred and sixty-eight newborns were enrolled within 15 days from birth. One hundred and fifty-five of them effectively entered the study and were randomized in probiotic and placebo group, receiving the formulation for 90 days. The probiotic formulation consists of a 1:1 mixture of 2 strains of $B$. breve prepared in an oily suspension and administered in a daily dosage of 5 drops containing $10^{8} \mathrm{CFU}$ of each strain. Absolute quantification of selected microbial groups in the faeces was performed using qPCR. Anthropometric data, daily diary minutes of crying, number of regurgitations, vomits and evacuations, and colour and consistency of stools were evaluated before and after treatment. The study confirmed the positive role of breast milk in influencing the counts of target microbial groups, in particular the bifidobacteria community. No adverse events upon probiotic administration were reported, suggesting the safety of the product in this regimen. $B$. breve counts increased significantly in all administered newborns $(p<0.02)$. The study demonstrates that a 3 months treatment with $B$. breve strains in healthy breast-fed newborns helps to prevent functional gastrointestinal disorders, in particular reducing $56 \%$ of daily vomit frequency $(p<0.03)$, decreasing $46.5 \%$ of daily evacuation over time $(p<0.03)$, and improving the stool consistency (type 6 at the Bristol Stool chart instead of type 5$)$ in those at term $(p<0.0001)$. Moreover, a significant reduction (8.65 vs. $7.98 \mathrm{LogCFU} / g$ of feces, $p<0.03$ ) of $B$. fragilis in the bottle-fed group receiving the probiotic formulation was observed.

Keywords: probiotic, Bifidobacterium breve, infant colic, functional gastrointestinal disorders, breastfeeding, bottle-feeding, microbiota 


\section{INTRODUCTION}

Infant colic is a common disorder in the first 3 months of childhood that affects up to $30 \%$ of newborns and is characterized by paroxysmal, excessive, incontrollable crying without identifiable causes (1). Wessel et al. (2) gave the first definition of this disorder as a condition of crying or fussing that lasts more than $3 \mathrm{~h}$ per day, more than 3 days per week. For a clinical purpose, the Rome IV consensus group (3) recently revised the diagnostic criteria including the age of newborn ( $<5$ months), giving less importance to the amount of crying considering instead the prolonged and unsoothable character of the crying episodes as well as irritability that cannot be prevented or resolved by caregivers. Symptoms, such as flushing of the face, meteorism, thighs flexion and flatulence, begin in the second week of life, in both breast-fed and formula-fed infants, and usually resolve spontaneously over time (4).

Infant colic represents a serious problem for the family, because caregivers have difficulties in dealing with these incontrollable crises often resulting in stress and concerns; a prospective European multicenter study carried out by Vik et al. (5) revealed that infantile colic and prolonged crying are associated with high maternal depression scores. Similarly, regurgitation, vomit and constipation frequently require a pediatrician visit during the first 6 months of life and are often responsible for feeding changes, and use of medical treatments $(6,7)$. Moreover, several consequences were associated to the presence of colics in the early stage of life: children with a history of colics have a higher prevalence of functional gastrointestinal disorders later in life (8) and children with migraine were more likely to have experienced infantile colic than those without migraine (7). Therefore, an effective preventive strategy against functional gastrointestinal disorders is envisaged.

Despite 40 years of research, the etiology of colic crises and other functional gastrointestinal disorders has not been fully clarified. It has been suggested that a number of behavioral factors (psychological and social), nutritional factors (food hypersensitivity or allergy), intestinal dysmotility and low grade intestinal inflammation can contribute to its occurrence $(6,9)$. Being a typical disorder of the gastrointestinal tract, it is not surprising that imbalance in the gut microbiota composition has been suggested to play a role in the pathogenesis of these conditions. The gut microbiota has a very close relation with the host contributing to the normal human physiology: it can provide a barrier for colonization of pathogens, synthesize vitamins, and other beneficial compounds and stimulate the immune system (10). The neonatal period is a crucial stage for gastrointestinal colonization, a balanced composition of the gut microbiota resulting in a positive effects on the host health (11). Colicky infants have a reduced fecal-bacterial diversity and stability, compared to the healthy ones. They also show a higher prevalence of gram negative bacteria, especially coliforms, and a reduced abundance of beneficial bacteria, such as lactobacilli and bifidobacteria (12).

Diet has a dominant role in shaping the gut microbiota, therefore the type of feeding in newborns has a certain impact on the assessment of the intestinal microbial groups. Remarkable differences were shown by Lee et al. (13): Actinobacteria was the predominant phylum in breastfed newborns, followed by Firmicutes and Proteobacteria; in contrast, in formulafed infants, the proportions of Actinobacteria and Firmicutes were similar, followed by Proteobacteria. In addition, the gut microbiota of formula-fed infants contains a significant amount of the genera Escherichia, Veillonella, Enterococcus, and Enterobacter, whereas the content of Lactobacillus was low. The same work reports that the main genus in both breastand formula-fed infants is Bifidobacterium, but the proportion resulted significantly higher in breastfed infants. The study of Mazzola et al. (14) also showed a reduced Bifidobacterium spp. count in mixed-fed infants (fed with at least 50\% formula milk) with respect to breastfed. On the contrary, a differential representation of the genus Bifidobacterium was not detected in breastfed infants compared to formula-fed, although differences in the gut microbiota were observed in the two groups (15). Moreover, these studies detected lower bacterial richness and diversity in breastfed, probably for the presence of unique oligosaccharides in breast milk, which serve as selective metabolic substrates for a limited number of gut microbes (16).

Feeding type, influencing the composition of intestinal microbiota, could play a significant role in the pathogenesis of infant colics although after the first year of life these differences are lost (17). A recent study focused on colicky and non-colicky formula-fed infants, performed using FISH as bacterial counting technique, revealed a lower concentration of total bacteria and a higher abundance of Enterobacteriaceae in colicky formula-fed infants (18).

Several studies support the use of probiotics as therapeutic or preventive agents against various diseases, in particular enteric disorders but also human pathology which are not apparently linked to the microbial gut composition, such as allergies and autoimmune diseases $(19,20)$. A treatment with probiotics, whose beneficial effects on the gut microbiota disorders and on human health are well known, may have a protective effect from gastrointestinal disorders including colics and reduce the symptoms associated, leading to a correct microbial colonization in early infancy, when the gut microbiota is still in a period of adjustment.

Many studies have focused on the administration of Lactobacillus reuteri DSM 17938 as probiotic for the prevention or reduction of symptoms of functional gastrointestinal disorders, including colic, regurgitation, vomit and constipation with successful results $(6,21,22)$. In particular, Savino et al. (23) evidenced a lower number of anaerobic gram negative bacteria, enterobacteriaceae and enterococci in colicky newborns that received $L$. reuteri compared to non treated babies. However, other Lactobacillus species, such as L. delbrueckii subsp. delbruekii DSM 20074 and L. plantarum MB 456, have shown inhibitory activity against gas-forming coliforms and they have the potential of being used in the management of infant colic (24). Differently, the administration of bifidobacteria for the treatment of these intestinal disorders remains scarcely investigated, although their role in the healthy newborn gut microbiota has been demonstrated as reviewed by Di Gioia et al. (25). A previous in vitro study described the capability of some strains belonging to Bifidobacterium genus, including Bifidobacterium breve strains, of inhibiting in vitro the growth 


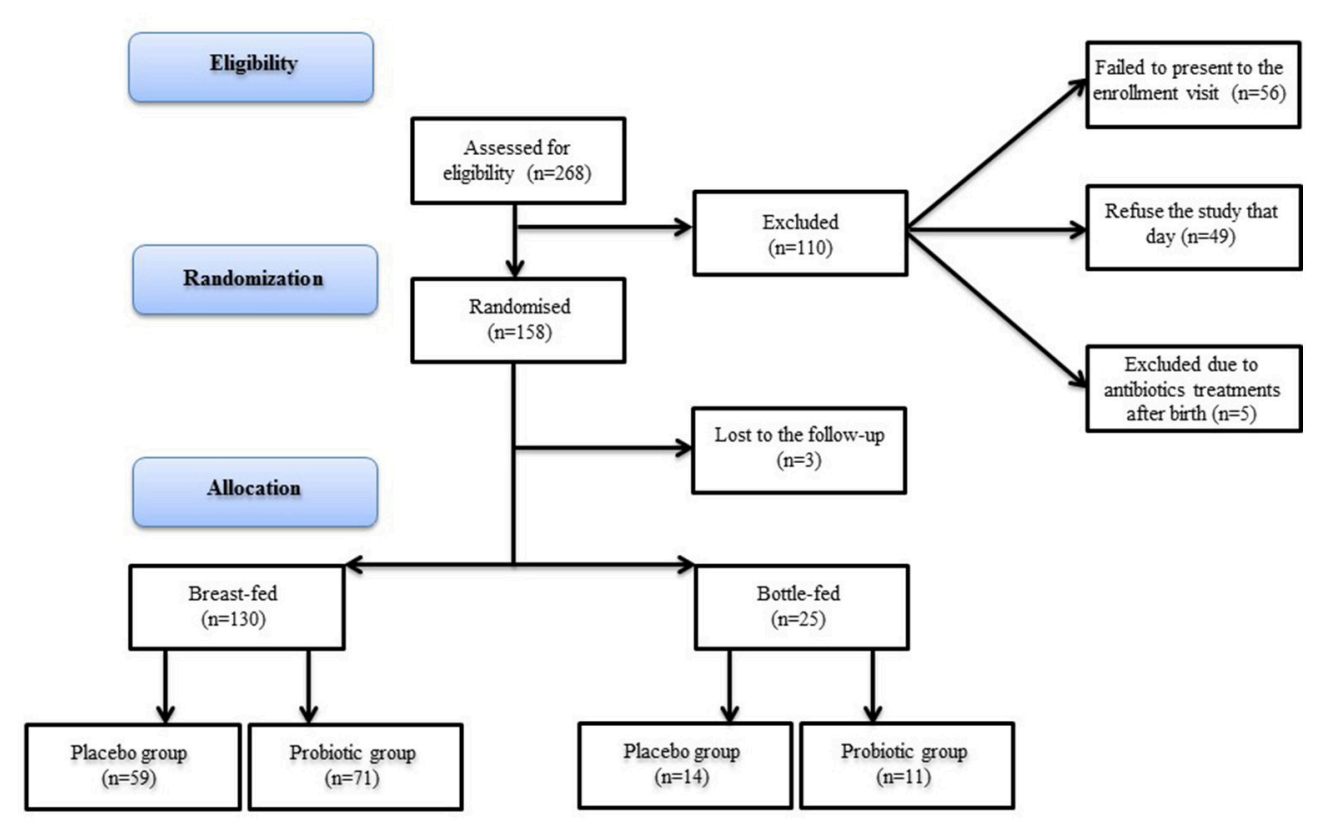

FIGURE 1 | Study flow diagram.

of pathogens typical of the infant gastrointestinal tract including coliforms isolated from colicky newborns (26). Other studies demonstrated the efficacy of $B$. breve, strains for the treatment of different infant diseases: Li et al. (27) showed the usefulness in promoting the colonization of $B$. breve and the formation of a normal intestinal biota in low birth weight infants, Wada et al. (28) described beneficial effects of this species in immunocompromised pediatric patients on chemotherapy. Moreover, recent studies have evidenced the effectiveness of $B$. breve to reduce the risk of necrotizing enterocolitis in preterm infants $(29,30)$. In addition, the two strains B. breve $\mathrm{B} 632$ and BR03 have been investigated for their capability of colonizing human intestine, stimulating the immune response, competing against pathogens and their safety assessments have been also demonstrated $(26,31,32)$. A recent study also showed the capability of these $B$. breve strains, used as probiotic for children with celiac disease, to act as a "trigger" element for the increase of other beneficial bacterial genus or phylum, like Firmicutes (33).

The aim of this study was to describe the effectiveness of a $B$. breve based probiotic formulation administered both to breast-fed and bottle-fed newborns in: 1) shifting the counts of targeted fecal microbial groups; 2) preventing colic symptoms and functional gastrointestinal disorders in a cohort of healthy newborns.

\section{MATERIALS AND METHODS}

\section{Study Design and Samples Collection}

This was a double-blind, randomized, placebo-controlled clinical trial (NCT03219931) approved by the Ethical Committee of the Maggiore della Carità Hospital (CE 63/13). The newborns were enrolled at the Department of Medical Sciences, Division of
Pediatrics, University of Piemonte Orientale “A. Avogadro" in a period from November 2013 to September 2016. Newborns were recruited at birth and enrolled within 15 days from birth during the first visit (T0). Informed consent was obtained by parents at the enrolment, in accordance with the local Ethics Committee and Helsinki criteria. Patients were asked to perform a second visit (T1) after 90 days of treatment. The number of newborns assessed for eligibility [268], randomized [155], and allocated to the placebo or probiotic group is shown in Figure 1. They were recruited if healthy within 15 days from birth and born adequate for gestational age. Exclusion criteria were: 1) twin neonates; 2) treatments with any type of drug within the enrolment; 3) treatments with probiotics; 4) smoking mothers; 5) family history for congenital diseases; 6) history of prolonged jaundice. No specific dietary restrictions during lactation were recommended to the mothers, with the exception of other products containing probiotics. Patients were randomized using a computer-generated allocation sequence in Placebo or Probiotic group (1:1). The study personnel and parents were masked to the study group allocation. The original idea of the study was to recruit an equal number of breast-fed and bottle-fed newborns but, considering the difficulties in the enrolment in bottle-fed ones, we decided to go on with a different number of newborns belonging to the two groups (Figure 1).

The Probiotic group received a commercial probiotic formulation Bifibaby ${ }^{\circledR}$ (Probiotical S.p.A., Novara, Italy) containing B. breve for 90 days (T1) and the Placebo group received a placebo formulation for the same period. Probiotic formulation was a 1:1 mixture of 2 strains, $B$. breve BR03 (DSM 16604) and B. breve B632 (DSM 24706) prepared in an oily suspension, administered in a daily dosage of 5 drops containing $10^{8} \mathrm{CFU}$ of each strain. Placebo was prepared with the same 
TABLE 1 | Primer sequences and qPCR conditions used in the different assays.

\begin{tabular}{|c|c|c|c|c|}
\hline Microorganism target & Primer & Sequence $\left(5^{\prime}-3^{\prime}\right)$ & Amplicon length (bp) & References \\
\hline \multirow[t]{2}{*}{ Escherichia coli } & Eco-F & GTTAATACCTTGGCTCATTGA & 340 & (34) \\
\hline & Eco-R & ACCAGGGTATCTAATCCTGTT & & \\
\hline \multirow[t]{2}{*}{ C. difficile } & Cdiff-F & TTGAGCGATTACTTCGGTAAAGA & 114 & (35) \\
\hline & Cdiff-R & TGTACTGGCTCACCTITGATATTCA & & \\
\hline \multirow[t]{2}{*}{ Bifidobacterium spp. } & Bif-F & TCGCGTCYGGTGTGAAAG & 243 & (36) \\
\hline & Bif-R & CCACATCCAGCRTCCAC & & \\
\hline \multirow[t]{2}{*}{ Lactobacillus spp. } & Lac-F & GCAGCAGTAGGGAATCTTCCA & 349 & (37) \\
\hline & Lac-R & GCATTYCACCGCTACACATG & & \\
\hline \multirow[t]{2}{*}{ Bacteroides fragilis group } & Bfra-F & CGGAGGATCCGAGCGTTA & 92 & (38) \\
\hline & Bfra-R & CCGCAAACTTCACAACTGACTTA & & \\
\hline \multirow[t]{3}{*}{ B. breve } & F_IS & GTGGTGGCTTGAGAACTGGAT AG & 118 & (39) \\
\hline & R_IS & CAAAACGATCGAAACAAACACTAAA & & \\
\hline & P_IS & TGATTCCTCGTTCTTGCTGT & & \\
\hline \multirow[t]{2}{*}{ Enterobacteria } & Ent-F & ATGGCTGTCGTCAGCTCGT & 385 & $(40)$ \\
\hline & Ent-R & ССТАСТTСТПТGСAАСССАСТС & & \\
\hline
\end{tabular}

excipients without probiotic strains using an identical form of package.

\section{Clinical Monitoring}

Delivery and birth data were collected during the first visit. Anthropometric data (weight, height, head circumference) and type of feeding information were collected at both the first (T0) and second visit (T1).

Parents were asked to record on a daily diary minutes of inconsolable crying according to a validated questionnaire (41). They also recorded daily number of regurgitations, vomits, and evacuations, and colour and consistency of stools. The Bristol Stool Form Scale for children was given to parents (42). Colic was diagnosed according to the Rome IV consensus group (3).

Parents were also asked to report any adverse event (in particular constipation, vomit, allergic reactions, illness), treatments, number and type of infections, or abdominal pain occurred during the trial period. The adherence was monitored by biweekly phone calls, counting empty vials, and checking daily dairies.

\section{Stool Samples Collection}

Faecal samples of newborns were collected twice, on enrolment (T0) and at the end of the intervention with probiotic/placebo (T1). The analyzed groups were therefore: Probiotic T0, Placebo T0, Probiotic T1, Placebo T1. Faecal samples were frozen immediately after collection at $-80^{\circ} \mathrm{C}$, in numbered screw-capped plastic containers, until they were processed for DNA extraction. Researchers performing DNA extraction and molecular analyses (qPCR) were blind to the group identity of patients (Probiotic or Placebo group).

\section{DNA Extraction From Faecal Samples}

DNA was extracted from $200 \mathrm{mg}$ of faeces (preserved at $-80^{\circ} \mathrm{C}$ after collection) using the QIAamp DNA Stool Mini Kit (Qiagen, West Sussex, UK) with a slight modification of the standard protocol: a supplementary incubation at $95^{\circ} \mathrm{C}$ for $10 \mathrm{~min}$ of the stool sample with the lysis buffer was added to enhance the bacterial cell rupture (43). Extracted DNA was stored at $-80^{\circ} \mathrm{C}$. The purity of DNA was determined by measuring the ratio of the absorbance at 260 and $280 \mathrm{~nm}$ (Infinite ${ }^{\circledR} 200$ PRO NanoQuant, Tecan, Mannedorf, Switzerland) and the concentration was evaluated by Qubit ${ }^{\circledR} 3.0$ Fluorometer (Invitrogen, Life Technologies, CA, USA).

\section{Absolute Quantification of Selected Microbial Groups Using Quantitative PCR (qPCR)}

Quantification of selected microbial groups or species usually monitored in studies related to infants $(38,43)$, i.e., Bidobacterium spp., Lactobacillus spp., Bacteroides fragilis group (comprising the most abundant species in human $B$. fragilis, B. distasonis, B. ovatus, B. thetaiotaomicron, B. vulgatus), B. breve, Clostridium difficile, Escherichia coli, and total enterobacteria, was performed with real-time PCR on DNA extracted from stool samples. The assays were carried out with a $20 \mu \mathrm{L}$ PCR amplification mixture containing $10 \mu \mathrm{L}$ of Fast SYBR ${ }^{\circledR}$ Green Master Mix (Applied Biosystems, Foster city, CA, USA) optimized concentrations of primers (Tables 1, 2), molecular grade $\mathrm{H}_{2} \mathrm{O}$ and $2 \mu \mathrm{L}$ DNA obtained from faecal samples at a concentration of $2.5 \mathrm{ng} / \mu \mathrm{L}$. B. breve analysis was performed using a TaqMan assay containing $12.5 \mu \mathrm{L}$ of Universal TaqMan master mix (Applied Biosystems, Foster city, CA, USA) $300 \mathrm{nM}$ 
TABLE 2 | qPCR amplification protocols and primer concentrations.

\begin{tabular}{|c|c|c|c|c|c|c|}
\hline Target bacteria & Initial denaturation & Denaturation & Annealing & N. cycles & Fw (nM) & $\operatorname{Rev}(\mathrm{nM})$ \\
\hline \multicolumn{7}{|l|}{ E. coli } \\
\hline Eco-F/Eco-R & $95^{\circ} \mathrm{C}-20 \mathrm{~s}$ & $95^{\circ} \mathrm{C}-3 \mathrm{~s}$ & $60^{\circ} \mathrm{C}-30 \mathrm{~s}$ & 40 & 400 & 400 \\
\hline \multicolumn{7}{|l|}{ C. difficile } \\
\hline Cdiff-F/Cdiff-R & $95^{\circ} \mathrm{C}-20 \mathrm{~s}$ & $95^{\circ} \mathrm{C}-3 \mathrm{~s}$ & $60^{\circ} \mathrm{C}-30 \mathrm{~s}$ & 40 & 250 & 250 \\
\hline \multicolumn{7}{|c|}{ Bifidobacterium spp. } \\
\hline BifTOT-F/BifTOT-R & $95^{\circ} \mathrm{C}-20 \mathrm{~s}$ & $95^{\circ} \mathrm{C}-3 \mathrm{~s}$ & $60^{\circ} \mathrm{C}-35 \mathrm{~s}$ & 40 & 200 & 300 \\
\hline \multicolumn{7}{|l|}{ Lactobacillus spp. } \\
\hline Lac-F/Lac-R & $95^{\circ} \mathrm{C}-20 \mathrm{~s}$ & $95^{\circ} \mathrm{C}-3 \mathrm{~s}$ & $63.5^{\circ} \mathrm{C}-30 \mathrm{~s}$ & 40 & 200 & 200 \\
\hline
\end{tabular}

of each primers and $100 \mathrm{nM}$ of probe labeled with the $5^{\prime}$ reporter dye 6-carboxyfluorescein and the $3^{\prime}$ quencher NFQ-MGB (Applied Biosystems, Nieuwerkerk a/d IJssel, The Netherlands). The number of PCR cycles was 40 .

The primer concentrations were optimized through primer optimization matrices in a 48 -well plate and estimating the best $\mathrm{Ct} / \Delta \mathrm{Rn}$ ratio. The different primers were also checked for their specificity utilizing the database similarity search program nucleotide-nucleotide BLAST (44). Moreover, to evaluate the specificity of amplification, analysis of product melting curve was performed after the last cycle of each amplification. The data obtained from the amplification were then converted to obtain the number of bacterial cells ( $\log \mathrm{CFU} / \mathrm{g}$ faeces) in accordance with the rRNA copy number available at the rRNA copy number database (45). Standard curves were constructed using $16 \mathrm{~S}$ rRNA PCR products of type strains of each target microorganism; the standard microorganisms used were $B$. breve $\mathrm{ReO} 2$, Lactobacillus plantarum ATCC 14917, B. fragilis DSM 2151, B. breve B632 DSM 20213, Clostridium sporogenes ATCC 319, E. coli ATCC 8739. PCR products were purified with a commercial DNA purification system (NucleoSpin ${ }^{\circledR}$ Extract II kit, MACHEREY-NAGEL GmbH \& Co. KG, Germany) and the concentration measured spectrophotometrically at $260 \mathrm{~nm}$. Serial dilutions were performed and $10^{2}, 10^{3}, 10^{4}, 10^{5}, 10^{6}, 10^{7}$ copies of the gene per reaction were used for calibration. Sample reactions were conducted in triplicate, with a negative control per each reaction.

\section{Statistical Analysis}

Data were expressed as mean \pm SD. Skewed variables were log transformed. Daily data were divided in 9 categories representing the mean of 10 consecutive days (from 0 to 90 day).

According to the primary outcome, a sample of 58 individuals per group has been estimated to be sufficient to demonstrate a difference between placebo and probiotics of $0.70 \mathrm{Log}$ CFU/g of bifidobacteria with a SD of 1.6 , a $90 \%$ power, and a significance level of $95 \%$, and a drop-out rate of $20 \%$ according to published data already available during the protocol design (32). According to the secondary outcome, a sample of 55 individuals per group has been evaluated sufficient to reduce of $30 \%$ the proportion of gastrointestinal disorders (colic, regurgitation, vomit, constipation) with an estimated prevalence of $40 \%$, according to literature $(1,6)$.
Data of microbial counts were subjected to Shapiro test and Bartlett test in order to verify the normal distribution of data and homogeneity of variances. The baseline characteristics were compared with a Fisher's exact test for categorical variables and a two sample $t$-test or the Welch's $t$-test when appropriate for continuous variables. A two-way repeated measure ANOVA was performed to evaluate the time effect, the treatment effect and the interaction effects (model 1) on the dependent variables (minutes of crying, stool characteristics, episodes of vomits and regurgitation, microbial counts). Sum of squares type III was used. The following covariates were also subsequently introduced: sex, type of delivery (vaginal, caesarean, operative), intrapartum antibiotic prophylaxis (IAP), gestational age, neonatal weight (model 2). Model 3 also included the type of feeding during the 90 days (breast-, bottle-, mixedfeeding). Furthermore, in model 2 and 3, weight, length, and head circumference were also corrected for the corresponding variable at birth. All the statistical analyses were performed using R Statistical Software and SPSS for Windows version 17.0 (SPSS Inc., Chicago, IL, USA).

\section{RESULTS}

\section{Baseline Characteristics of Enrolled Newborns}

At birth, 268 newborns were assessed for eligibility and their parents accepted the study. One hundred and ten did not enter the study because failed to present to the enrollment visit (46), refused the study that day (47) or were excluded due to antibiotic treatments after birth (5). The 158 subjects were assigned randomly to placebo or probiotic group. Three of them were lost at the follow-up and were excluded (Figure 1). Of the 155 newborns who entered in the protocol, 130 were breast-fed (59 placebo, 71 probiotics) and 25 were bottle-fed (14 placebo, 11 probiotics). Eighty-one were males, and 74 females. Moreover, 139 neonates were born by vaginal, 10 by cesarean, and 6 from operative delivery. All the enrolled mothers were healthy without suffering of chronic diseases. Fifteen mothers received IAP. Three mothers have an episode of flu during the study (2 subjects in the breastfeeding group and 1 subject in the formula feeding group). No mother was treated with antibiotics during lactation.

Table 3 represents clinical data and microbiological fecal counts at baseline in the two groups of allocation (placebo and 
TABLE 3 | Auxological characteristics of the whole cohort at baseline (T0) according to the allocation treatment.

\begin{tabular}{lcc}
\hline & Placebo & Probiotic \\
\hline Gender (M/F) & $34 / 39$ & $47 / 35$ \\
Gestational age (weeks) & $39.1 \pm 1.2$ & $39.3 \pm 1.0$ \\
Neonatal weight (g) & $3307.9 \pm 397.5$ & $3298.5 \pm 362.7$ \\
Length (cm) & $50.1 \pm 2.0$ & $50.1 \pm 1.7$ \\
Head circumference (cm) & $34.2 \pm 1.4$ & $33.9 \pm 1.1$ \\
Delivery (V/C/O) & $63 / 7 / 3$ & $76 / 3 / 3$ \\
Days of life & $10.6 \pm 1.9$ & $10.4 \pm 2.4$ \\
Breast-/Bottle-feeding & $59 / 14$ & $71 / 11$ \\
Daily crying (min) & $25.5 \pm 28.8$ & $28.8 \pm 37.7$ \\
Stool frequency & $3.8 \pm 1.8$ & $4.1 \pm 1.9$ \\
Regurgitation episodes & $1.7 \pm 1.8$ & $1.6 \pm 1.4$ \\
Vomit episodes & $0.1 \pm 0.2$ & $0.2 \pm 0.4$ \\
\hline
\end{tabular}

Data are expressed as mean $\pm \mathrm{SD}$. C, cesarean; O, operative; $V$, vaginal.

TABLE 4 | Mean counts (Log CFU/g of faeces) of different microbial groups analyzed in stool samples of the whole cohort.

\begin{tabular}{lcccc}
\hline Target & Probiotic T0 & Probiotic T1 & Placebo T0 & Placebo T1 \\
\hline Bifidobacterium spp. & $7.00 \pm 1.41$ & $7.51 \pm 0.88$ & $6.88 \pm 1.14$ & $7.29 \pm 1.06$ \\
B. breve & $4.45 \pm 1.85$ & $6.40 \pm 1.31^{*}$ & $4.54 \pm 1.5$ & $5.33 \pm 1.5$ \\
Enterobacteria & $6.54 \pm 1.23$ & $6.38 \pm 1.14$ & $6.02 \pm 1.4$ & $6.5 \pm 1.02$ \\
E. coli & $6.72 \pm 1.93$ & $7.2 \pm 1.36$ & $6.35 \pm 2.07$ & $7.42 \pm 1.24$ \\
Lactobacillus spp. & $6.56 \pm 1.28$ & $5.60 \pm 1.23$ & $6.22 \pm 1.06$ & $5.28 \pm 1.48$ \\
B. fragilis group & $7.44 \pm 2.14$ & $7.62 \pm 1.97$ & $6.79 \pm 2.19$ & $7.23 \pm 2.00$ \\
C. difficile & $2.66 \pm 1.48$ & $2.82 \pm 1.50$ & $2.60 \pm 1.32$ & $3.06 \pm 1.58$ \\
\hline
\end{tabular}

${ }^{*}$ Significant changes at t-test $(p<0.02)$.

probiotic neonates). Only Lactobacillus spp. counts were higher in the placebo group than in the probiotic group at baseline.

Because it is well-known that feeding modulates gut microbial composition as well as clinical presentation also in neonates, we investigated if breast- and bottle-fed babies were different at baseline. Supplementary Table 1 represents clinical data and microbiological fecal counts at baseline in the two groups (breast fed and bottle-fed neonates). Crying time $(p<0.05)$ and stool frequency were higher $(p<0.04)$ and regurgitation episodes were less frequent $(p<0.05)$ in breastfed infants. Total enterobacteria $(p<0.004)$, E. coli $(p<0.03)$, and B. fragilis group $(p<0.01)$ counts were lower in breastfed than in bottle-fed newborns, also when corrected for confounders (sex, gestational age, neonatal weight, type of delivery, IAP, and days of life at the entry date).

\section{Microbiological Results in Whole Cohort After Probiotic and Placebo Administration}

Table 4 shows the average crude microbial count obtained from the two groups of samples: probiotic and placebo. This analysis showed a significant increase of $B$. breve counts after 3 months. The other microbial groups did not show any significant difference.
Following this first evaluation and considering the different sample dimension of breast- and bottle-fed newborns as well as differences of baseline microbial counts in these two groups, an analysis separating breastfed from bottle-fed newborns was carried out.

\section{Data Evaluation of Probiotic Treatment on Breastfed Newborns}

At baseline, the placebo group had less stool frequency $(p<0.03)$ and lower enterobacteria counts than the probiotic one $(p<0.01)$, also when corrected for confounders (sex, gestational age, neonatal weight, type of delivery, and days of life at the entry date). The marginal means for stool frequency and enterobacteria counts are: $3.2 \pm 0.4$ vs. $4.3 \pm 0.3(p<0.01)$, and $5.86 \pm 0.36$ vs. $6.01 \pm 0.38 \mathrm{Log}$ CFU/g, $(p<0.03)$, respectively.

The compliance to the treatment was high. All the parents answered to the phone calls. $98.7 \%$ of them brought back correctly the empty vials. No adverse events were reported. Three infants ( 1 in placebo and 2 in probiotic) received simethicone for several days ( $<30$ days) due to regurgitations.

After 90 days, 100 (43 in placebo, 57 in probiotics) of the 130 neonates were still breastfed. In the remaining 30 infants, 7 were bottle-fed ( 5 in placebo, 2 in probiotic), and the other 23 were in mixed feeding (11 in placebo, 12 in probiotic).

\section{Clinical Data}

Considering clinical data, minutes of crying $(p<0.005)$ decreased over time without an effect of treatment also in the corrected models.

The number of the evacuation decreased over time $(p<0.0001)$, with an effect of treatment in those born after 40 weeks of gestational age $(p<0.03)$. Stool consistency was more type 5 at the Bristol Stool Chart $(p<0.03)$ over time. Children born after 40 weeks of gestational age had more frequently type 6 at the Bristol Stool Chart in those in probiotics $(p<0.0001)$.

Episodes of regurgitation decreased constantly over time $(p<0.01)$ in the probiotic group diversely by placebo group accordingly to different type of feeding ( $p<0.03$, Figure 2$)$.

Episodes of vomits decreased significantly with time in the probiotic group but not in the placebo group $(p<0.03)$. Moreover, during the 90 days the prevalence of colic infants was similar in the placebo (4 subjects, $6.8 \%$ ) and probiotic group (6 subjects, $8.5 \%$ ).

Interestingly, also auxological variables were modified. Infants in probiotics had a lower increase in weight during the study in those born with a cesarean delivery ( $p<0.03$; Figure 3 ), and in those still breastfed or switched to bottle-fed during the study $(p<0.005)$. Diversely, infants in probiotics had a higher increase in head circumference in those bottle-fed or with a mixed-feeding $(p<0.01)$.

Table 5 describes marginal means of model 1. Supplementary Table 2 describes also corrected models.

\section{Microbial Data}

Total Enterobacteria $(p<0.005)$, Bifidobacterium spp. $(p<0.001)$ and E. coli $(p<0.001)$ changed within time, 


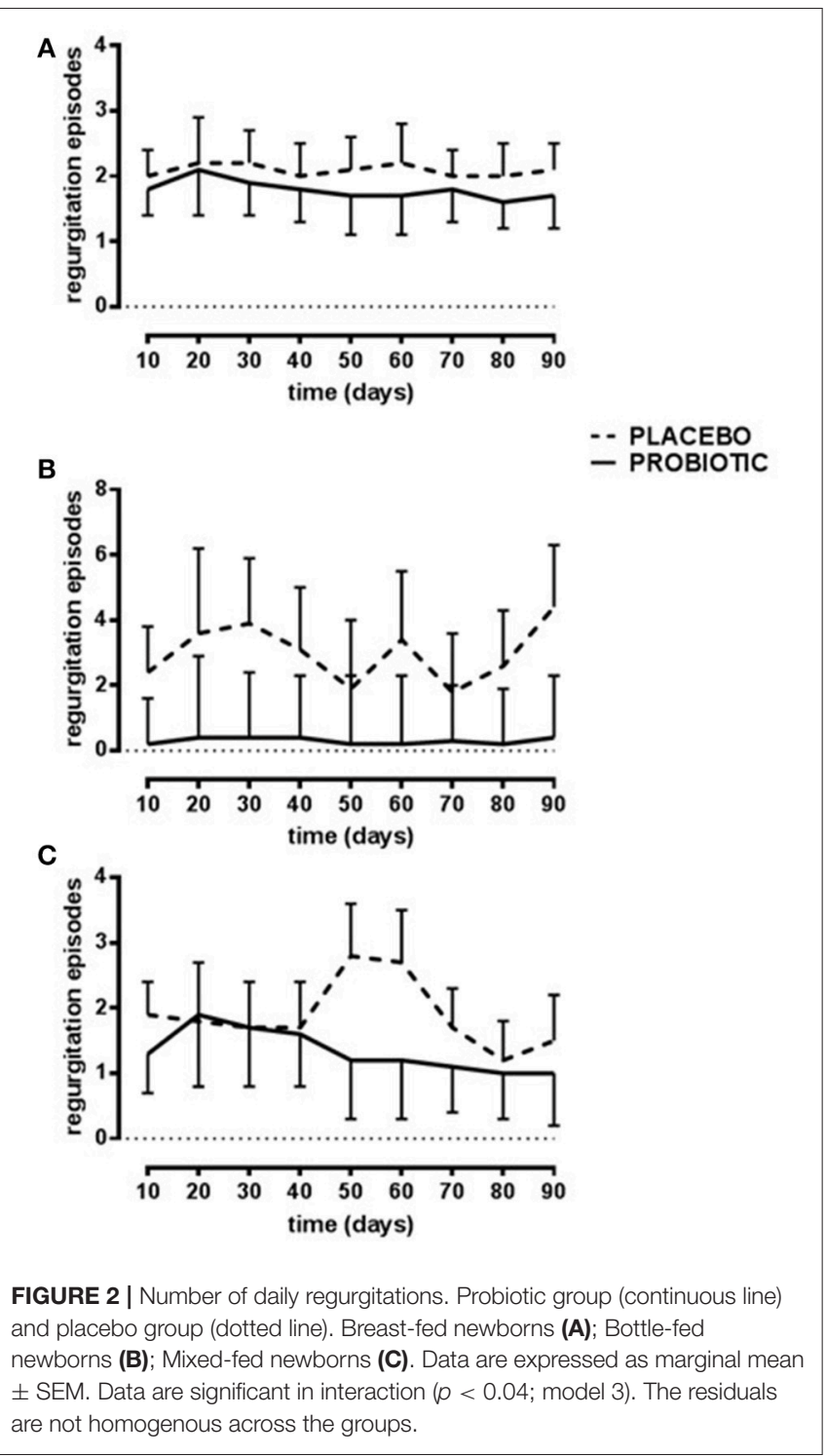

but the significance was lost when corrected for confounders. $C$. difficile did not change.

In those treated with probiotics, $B$. fragilis group members decreased within time in those born vaginally, whereas increased in the other infants $(p<0.04)$. Moreover, $B$. breve increased within time in those treated with the probiotics $(p<0.04)$. Microbiological data are reported in Table 6.

\section{Data Evaluation of Probiotic Treatment on Bottle-Fed Newborns}

At baseline, placebo group had less Lactobacillus spp. counts than probiotic one $(p<0.008)$. When corrected for confounders (sex, gestational age, neonatal weight, and days of life at the entry date), the statistical significance was lost, whereas $B$. fragilis group counts were higher in the probiotic group (marginal means are $6.62 \pm 0.53$ vs. $8.62 \pm 0.67 \mathrm{Log} \mathrm{CFU} / g, p<0.02)$.

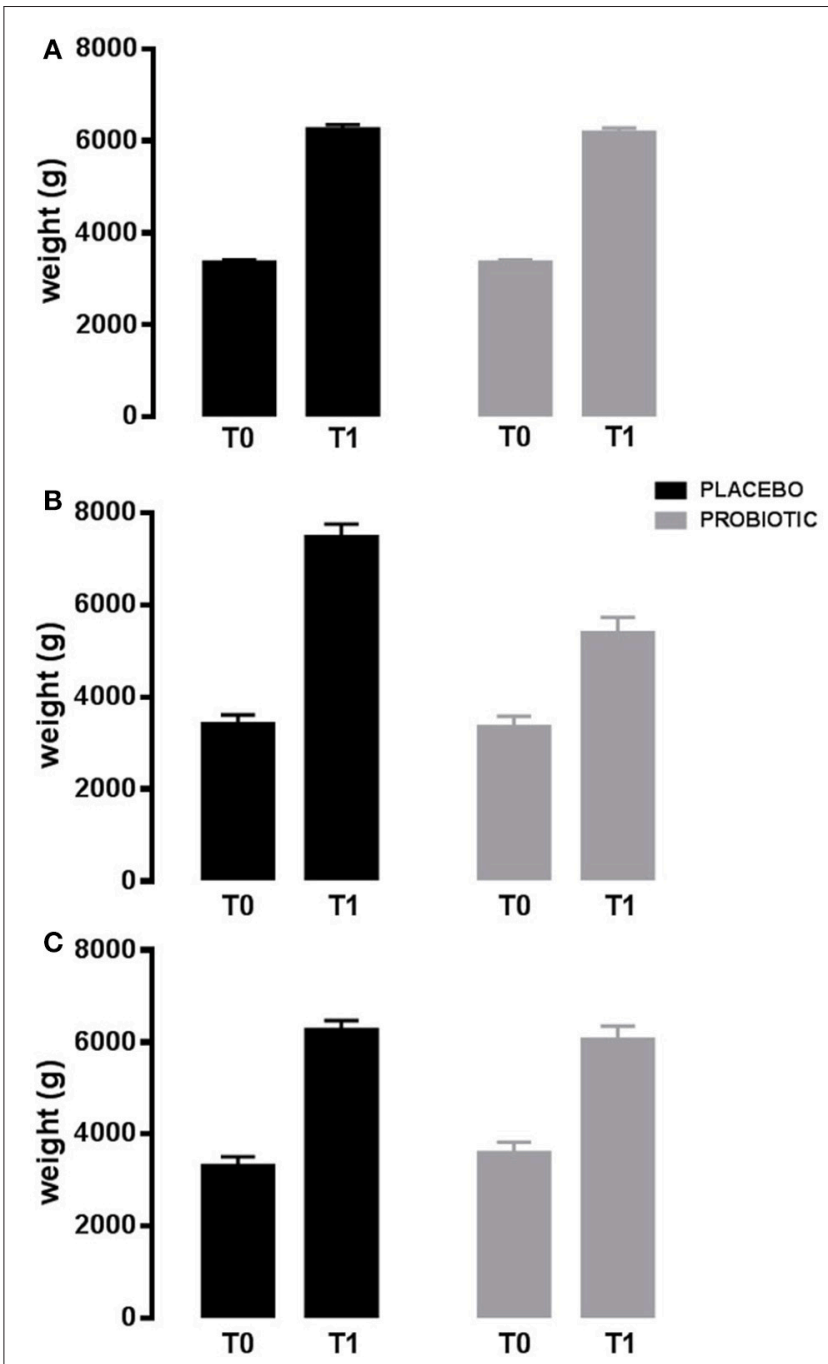

FIGURE 3 | Weight variation in 90 days. Probiotic group (gray bar) and placebo group (black bar). Newborns born by vaginal delivery (A); Newborns born by cesarean delivery (B); Newborns born by operative delivery (C). Data are expressed as marginal mean \pm SEM. Data are significant in interaction $(p<0.03$; model 2). T0: baseline. T1: after 90 days of placebo/probiotic.

\section{Clinical Data}

Considering clinical data in both crude and corrected analysis, no changes were detected in minutes of crying, stool frequency and consistency, episodes of vomits or regurgitations. No infants had colic in both groups. Weight $(p<0.0006)$, length $(p<0.01)$, and head circumference $(p<0.005)$ increased with time without an effect of the treatment.

\section{Microbial Data}

Total enterobacteria and $E$. coli did not change over time. Bifidobacterium spp. $(p<0.02)$ and $C$. difficile increased $(p<0.04)$ with time without a treatment's effect. In those treated with probiotics, $B$. fragilis group $(p<0.03)$ decreased and $B$. breve increased $(p<0.03)$, respectively with time. Microbiological data are reported in Table 7 . 
TABLE 5 | Clinical and anthropometric variations in the breast-fed group obtained with multivariable analysis of repeated measure.

\begin{tabular}{lcccc}
\hline Target & Probiotic T0 & Probiotic T1 & Placebo To & Placebo T1 \\
\hline Crying(min) & $25.4 \pm 4.5$ & $19.3 \pm 2.9$ & $32.1 \pm 5.6$ & $20.5 \pm 3.7$ \\
Stool frequency & $4.3 \pm 0.2$ & $2.1 \pm 0.1$ & $3.9 \pm 0.2$ & $2.0 \pm 0.1$ \\
Stool color & $6.0 \pm 0.1$ & $5.7 \pm 0.1$ & $6.0 \pm 0.1$ & $6.0 \pm 0.1$ \\
Stool consistency & $5.8 \pm 0.1$ & $5.7 \pm 0.1$ & $6.0 \pm 0.1$ & $5.6 \pm 0.1$ \\
Regurgitations & $2.0 \pm 0.2$ & $1.6 \pm 0.2$ & $1.8 \pm 0.2$ & $2.0 \pm 0.3$ \\
Vomits & $0.25 \pm 0.05$ & $0.11 \pm 0.06^{\star}$ & $0.10 \pm 0.05$ & $0.20 \pm 0.07$ \\
Weight (g) & $3310.9 \pm 43.7$ & $3465.6 \pm 57.4$ & $3321.5 \pm 48.0$ & $3452.2 \pm 62.9$ \\
Length (cm) & $50.1 \pm 0.2$ & $51.5 \pm 0.2$ & $50.2 \pm 0.2$ & $51.5 \pm 0.2$ \\
HC (cm) & $33.9 \pm 0.1$ & $34.9 \pm 0.1$ & $34.3 \pm 0.1$ & $35.0 \pm 0.1$ \\
& & & &
\end{tabular}

All values are expressed as marginal means \pm standard error. HC, head circumference. *Significance in interaction (time * treatment) effect $(p<0.03)$.

TABLE 6 | Mean counts (Log CFU/g of faeces) of different microbial groups analyzed in stool samples of breast-fed newborns.

\begin{tabular}{lcccc}
\hline Target & Probiotic T0 & Probiotic T1 & Placebo T0 & Placebo T1 \\
\hline Bifidobacterium spp. & $7.11 \pm 0.38$ & $7.91 \pm 0.27$ & $6.59 \pm 0.34$ & $7.78 \pm 0.24$ \\
B. breve & $4.64 \pm 0.50$ & $6.10 \pm 0.47^{\star}$ & $4.40 \pm 0.48$ & $5.86 \pm 0.46$ \\
Enterobacteria & $6.01 \pm 0.38$ & $6.55 \pm 0.35$ & $5.86 \pm 0.36$ & $6.46 \pm 0.34$ \\
E. coli & $6.70 \pm 0.57$ & $7.46 \pm 0.51$ & $6.40 \pm 0.54$ & $7.06 \pm 0.48$ \\
Lactobacillus spp. & $6.22 \pm 0.33$ & $5.55 \pm 0.33$ & $6.28 \pm 0.29$ & $6.27 \pm 0.35$ \\
B. fragilis group & $6.34 \pm 0.64$ & $7.33 \pm 0.61$ & $6.31 \pm 0.61$ & $6.45 \pm 0.58$ \\
C. difficile & $2.70 \pm 0.42$ & $2.89 \pm 0.48$ & $2.82 \pm 0.37$ & $3.01 \pm 0.42$
\end{tabular}

All values are expressed as marginal means \pm standard error. *Significance in interaction (time * treatment) effect $(p<0.04)$.

TABLE 7 | Mean counts (Log CFU/g of faces) of different microbial groups analyzed in stool samples of bottle-fed newborns.

\begin{tabular}{lcccc}
\hline Target & Probiotic T0 & Probiotic T1 & Placebo T0 & Placebo T1 \\
\hline Bifidobacterium spp. & $6.80 \pm 0.36$ & $7.56 \pm 0.36$ & $6.79 \pm 0.30$ & $7.35 \pm 0.30$ \\
B. breve & $4.20 \pm 0.35$ & $6.42 \pm 0.37^{\star}$ & $4.11 \pm 0.31$ & $5.09 \pm 0.33$ \\
Enterobacteria & $7.30 \pm 0.41$ & $6.97 \pm 0.34$ & $6.75 \pm 0.36$ & $7.14 \pm 0.30$ \\
E. coli & $7.91 \pm 0.95$ & $7.38 \pm 0.62$ & $6.67 \pm 0.66$ & $7.51 \pm 0.54$ \\
Lactobacillus spp. & $6.81 \pm 0.29$ & $6.00 \pm 0.35$ & $6.05 \pm 0.26$ & $6.39 \pm 0.31$ \\
B. fragilis group & $8.65 \pm 0.61$ & $7.98 \pm 1.84^{*}$ & $7.56 \pm 0.56$ & $8.33 \pm 0.51$ \\
C. difficile & $2.54 \pm 0.44$ & $3.16 \pm 0.52$ & $2.59 \pm 0.39$ & $3.35 \pm 0.46$
\end{tabular}

All values are expressed as marginal means \pm standard error. *Significance in interaction (time * treatment) effect $(p<0.03)$.

\section{DISCUSSION}

The use of bifidobacteria as probiotics in infants is established for some enteric diseases, the most common of which is diarrhea (25). However, although in vitro studies support the use of bifidobacteria against gas-forming coliforms (26), no clinical trials have been performed up to now on their use against infant colics. This work was focused on the evaluation of the effects on functional gastrointestinal symptoms, including colics, of integration of the infant diet with a $B$. breve based probiotic formulation.

The study has clearly shown the capability of the administered $B$. breve strains to survive to the gastric transit and to reach the neonatal intestine. In fact, although $B$. breve was detected in all fecal samples, a significant increase was shown upon strain administration. In agreement with Lee et al. (13), a reduction of Lactobacillus counts was observed in all groups of newborns over time and this is particularly evident in the probiotic treated group. This could be related to a high ability of Bifidobacterium spp. to influence gut microbiota composition, by enhancing the blooming of some species and reducing others, as observed in other studies regarding Bifidobacterium administration (25).

Feeding type is known to have a crucial role in shaping the infant intestinal microbiota $(25,48)$. Our study shows that, at the enrolment, when 7-15 days of breast- or bottle-feeding had already been done, some differences were present in the groups with different feeding type: total enterobacteria and E. coli counts were higher in bottle-fed than in breast-fed newborns, also when corrected for confounders. In addition, higher counts of $B$. fragilis were found in bottle-fed infants at the baseline, in agreement with the higher risk of infection generally observed in non breast-fed infants (49). This higher count was also evident after treatment, both in the probiotic and the placebo group, confirming the absolute importance of the starting feeding type in shaping the gut microbiota and, in particular, in reducing gramnegative bacteria amount. However, in bottle-fed infants, the mean counts of $B$. fragilis were higher at the end of the treatment in the placebo group with respect to the probiotic one, thus indicating a possible positive effect of the $B$. breve administration at least before weaning. The increase of $B$. breve is also observed in breast-fed newborns not treated with probiotics and this, as already mentioned before, once more highlights the positive role of breast milk in shaping the bifidobacteria community, also considering that $B$. breve is one of the most abundant species in the newborn gut (50). This increase is also supported by the presence of peptides and oligosaccharides in the human milk that provide the stimulation of the growth of bifidobacteria (47).

In addition to the microbial data, this study aims at monitoring the typical gastrointestinal symptoms of colics, i.e., regurgitation, vomit and constipation, all of them difficultto-handle problems for caregivers. Results obtained from the applied models showed a decreased number of evacuations and an enhancement of stool consistency in breast-fed newborns after 90 days of probiotics. In addition, bottle-fed newborns showed an improvement of stool color. These data suggested an amelioration in the gastrointestinal transit which can be attributed to the probiotic intake. Moreover, the number of regurgitations and episodes of vomit was reduced after probiotic treatment. Similar results have already been demonstrated with a supplementation of $L$. reuteri (6). The reduction of these symptoms is particularly important because they also reduce parental anxiety and related consequences.

The reduction in regurgitation and vomit was not shown in the group of bottle-fed newborns. This result can be affected by the small size of the bottle-fed group of newborns. The study was not designed to evaluate differences between the two feeding regimens and authors are aware that the bottle-fed group was underpowered to reach the clinical outcomes. However, data related to the different feeding should be analyzed separately due to the unexpected significant differences in microbial 
composition at baseline. On the other hand, those breast-fed at the recruitment who switched to bottle-or mixed-feeding had an improvement with reduction of regurgitation episodes. This is an important achievement also considering that the number of newborns bottle-fed since the beginning of life is generally low as, usually, a starting feeding with mother milk is applied (51).

In this study, daily infant crying time did not show any difference between probiotic and placebo groups in spite of the improvement of the gastric transit due to the probiotic administration. This result is contrasting with other reports in literature. Several causes could be considered, first of all the inaccuracy of the count of minutes of crying through self-report diaries, although validated, in particular for such a prolonged time. Analysis of any existing tool to monitor daily crying have been demonstrated to be inaccurate, difficult or not validated for a prolonged time of observation (52). Moreover, we evaluated the effects over 3 months, whereas the majority of the studies are related to probiotic treatment no longer than 4 weeks (1, 53). Moreover, other confounding factors may have a role after the second months of life, in particular, if we consider the efficacy on the other gastro-intestinal parameters. Furthermore, the prevalence of colic infants was similar but very low in both placebo and probiotic groups. This is a consequence of considering in the study healthy newborns. Studies including only colic infants are needed in the future.

The main unexpected and interesting result of the study was related to the auxological parameters. Clinical trials on the effect of probiotics on neonatal growth parameters are scarce. In our population, infants born by cesarean section had a lower catchup growth in weight if treated with the probiotic. This result is of crucial interest in planning further intervention studies. Gut colonization by environmental microorganisms occurs during or immediately after the birth, whereas, in infants delivered by cesarean section, gut colonization is delayed and often altered, in particular modifying Bifidobacterium and Lactobacillus counts (25). Increasing epidemiological data suggested that children born by cesarean section have an increased risk to develop obesity later in life $(54,55)$. How the genetic background and the environment affect mechanisms that control appetite, weight regulation and metabolic disorders linked to overweight, and the immune education is poorly understood. Gestation, delivery, postnatal nutrition (lactation and weaning) have been identified as critical periods to program the nutritional and hormonal control of the offspring. Some Authors suggest that the sudden modification of the initial conditions may disrupt the physiological process predisposing to certain diseases $(46,56,57)$ and alterations in the precocious colonization have a role $(54,55)$. Our data suggest that a treatment with $B$. breve strains in the first 3 months of life is able to influence the microbiota composition and this is associated with a concomitant lower weight gain in the population at higher risk of metabolic disturbances in later life. Other authors failed to show changes in weight in neonates treated with other probiotics $(53,58)$. Differences should be secondary to the strains or, more probably, to the timing of the treatment being our protocol designed on 3 months, differently from the majority of the studies which followed infants for 1 month. In our study, the effect on weight was associated to an increase in head circumference. These data suggest that the probiotic treatment protects against a growth failure, as recently demonstrated for a multi-strain probiotic containing bifidobacteria in very low birth weight children exposed to antibiotics (59).

The main limitation of this study is related to the small sample size of the bottle-fed population. However, this is a consequence of the inclusion criteria (healthy neonates) in a condition in which breastfeeding must be the first choice (51). Furthermore, although we used a validated questionnaire for daily infant crying, the other questionnaires used to record other gastrointestinal symptoms are not validated. On the contrary, the strengths of our study are a treatment prolonged for more than 4 weeks, the inclusion of neonates not exposed to antibiotics, the evaluation of many confounders, in particular regarding birth and changes in feeding over time.

In conclusion, our study demonstrates that the administered $B$. breve strains can reach the intestine of healthy newborns, preventing functional gastrointestinal disorders and reducing the precocious weight gain, at least in the absence of antibiotic interferences. No adverse events were reported, suggesting the safety of the product in this regimen. Prospective longitudinal evaluations should be useful to further investigate if a precocious short treatment in this critical window has also advantages later in life.

\section{AUTHOR CONTRIBUTIONS}

DD and GB conceived and designed the study. EG and AS were responsible for patients recruitment and collection of clinical data. IA and NB performed the qPCR experiments and contributed to the writing of the paper. FP performed the statistical analysis, interpreted the results, and contributed to the writing of the paper. $\mathrm{DD}, \mathrm{SB}, \mathrm{LB}$, and FP critically revised and approved the final manuscript. LM and MP designed the probiotic supplement.

\section{ACKNOWLEDGMENTS}

The authors would like to thank Roberta Ricotti, Elisabetta Mingoia, Emanuele Mones, Francesca Archero, and Mauro Zaffaroni for their technical support. This paper is dedicated to Dr. Giovanni Mogna who strongly supported the described research.

\section{SUPPLEMENTARY MATERIAL}

The Supplementary Material for this article can be found online at: https://www.frontiersin.org/articles/10.3389/fnut.2018. 00039/full\#supplementary-material 


\section{REFERENCES}

1. Savino F, Pelle E, Palumeri E, Oggero R, Miniero R. Lactobacillus reuteri (American Type Culture Collection Strain 55730) versus simethicone in the treatment of infantile colic: a prospective randomized study. Pediatrics (2007) 119:e124-30. doi: 10.1542/peds.2006-1222

2. Wessel MA, Cobb JC, Jackson EB, Harris GSJ, Detwiler AC. Paroxysmal fussing in infancy, sometimes called "colic." Pediatrics (1954) 14:421-35.

3. Zeevenhooven J, Koppen IJN, Benninga MA. The New Rome IV criteria for functional gastrointestinal disorders in infants and toddlers. Pediatr Gastroenterol Hepatol Nutr. (2017) 20:1-13. doi: 10.5223/pghn.2017.20.1.1

4. Lucas A, Roberts ISJ. Crying, fussing and colic behaviour in breast- and bottle-fed infants. Early Hum Dev. (1998) 53:9-18. doi: 10.1016/S0378-3782(98)00032-2

5. Vik T, Grote V, Escribano J, Socha J, Verduci E, Fritsch M, et al. Infantile colic, prolonged crying and maternal postnatal depression. Acta Paediatr. (2009) 98:1344-8. doi: 10.1111/j.1651-2227.2009.01317.x

6. Indrio F, Di Mauro A, Riezzo G, Civardi E, Intini C, Corvaglia L, et al. Prophylactic use of a probiotic in the prevention of colic, regurgitation, and functional constipation a randomized clinical trial. JAMA Pediatr. (2014) 168:228-33. doi: 10.1001/jamapediatrics.2013.4367

7. Romanello S, Spiri D, Marcuzzi E, Zanin A, Boizeau P, et al. Association between childhood migraine and and history of infantile colic. JAMA J Am Med Assoc. (2013) 309:1607-12. doi: 10.1001/jama.2013.747

8. Indrio F, Mauro A Di, Riezzo G, Cavallo L, Francavilla R. Infantile colic, regurgitation, and constipation: an early traumatic insult in the development of functional gastrointestinal disorders in children? Eur J Pediatr. (2015) 174:841-2. doi: 10.1186/1824-7288-39-15

9. Banks S, Thomas M, Gordon M, Wallace C, Akobeng A. Probiotics to prevent infantile colic. Cochrane Database Syst Rev. (2016) 12:1-19. doi: 10.1002/14651858.CD012473

10. Candela M, Biagi E, Maccaferri S, Turroni S, Brigidi P. Intestinal microbiota is a plastic factor responding to environmental changes. Trends Microbiol. (2012) 20:385-91. doi: 10.1016/j.tim.2012.05.003

11. Sekirov I, Russell SL, Antunes LCM, Finlay BB. Gut microbiota in health and disease. Physiol Rev. (2010) 90:859-904. doi: 10.1152/physrev.00045.2009.

12. Dubois NE, Gregory KE. Characterizing the intestinal microbiome in infantile colic: findings based on an integrative review of the literature. Biol Res Nurs. (2016) 18:307-15. doi: 10.1177/1099800415620840

13. Lee SA, Lim JY, Kim B, Cho SJ, Kim NY, Bin Kim O, Kim Y. Comparison of the gut microbiota profile in breast-fed and formula-fed Korean infants using pyrosequencing. Nutr Res Pract. (2015) 9:242-8. doi: 10.4162/nrp.2015.9.3.242

14. Mazzola G, Murphy K, Ross RP, Di Gioia D, Biavati B. Early gut microbiota perturbations following intrapartum antibiotic prophylaxis to prevent group B Streptococcal disease. PLoS ONE (2016) 11:e0157527. doi: 10.1371/journal.pone.0157527

15. Azad MB, Mph TK, Maughan H, Guttman DS, Field CJ, Chari RS, et al. Gut microbiota of healthy Canadian infants: profiles by mode of delivery and infant diet at 4 months. CMAJ (2013) 185:385-94. doi: 10.1503/cmaj.130147

16. Abrahamsson TR, Jakobsson HE, Andersson AF, Engstrand L, Jenmalm MC. Low diversity of the gut microbiota in infants with atopic eczema. J Allergy Clin Immunol. (2005) 129:434-40. doi: 10.1016/j.jaci.2011.10.025

17. Guaraldi F, Salvatori G. Effect of breast and formula feeding on gut microbiota shaping in newborns. Front Cell Infect Microbiol. (2012) 2:94. doi: $10.3389 /$ fcimb.2012.00094

18. Savino F, Quartieri A, De Marco A, Garro M, Amaretti A, Raimondi S, et al. Comparison of formula-fed infants with and without colic revealed significant differences in total bacteria, Enterobacteriaceae and faecal ammonia. Acta Paediatr. (2017) 106:573-8. doi: 10.1111/apa.13642

19. Taylor AL, Hale J, Wiltschut J, Lehmann H, Dunstan JA, Prescott SL. Effects of probiotic supplementation for the first 6 months of life on allergenand vaccine-specific immune responses. Clin Exp Allergy (2006) 36:1227-35. doi: 10.1111/j.1365-2222.2006.02553.x

20. Roessler A, Friedrich U, Vogelsang H, Bauer A, Kaatz M, Hipler UC, et al. The immune system in healthy adults and patients with atopic dermatitis seems to be affected differently by a probiotic intervention. Clin Exp Allergy (2007) 38:93-102. doi: 10.1111/j.1365-2222.2007.02876.x
21. Szajewska H, Gyrczuk E, Horvath A. Lactobacillus reuteri DSM 17938 for the management of infantile colic in breastfed infants: a randomized, double-blind, placebo-controlled trial. J Pediatr. (2013) 162:257-62. doi: $10.1016 /$ j.jpeds.2012.08.004

22. Chau K, Lau E, Greenberg S, Jacobson S, Yazdani-brojeni P, Verma N, et al Probiotics for infantile colic: a randomized, double-blind, placebo-controlled trial investigating Lactobacillus reuteri DSM 17938. J Pediatr. (2015) 166:748.e1. doi: 10.1016/j.jpeds.2014.09.020

23. Savino F, Fornasero S, Ceratto S, De Marco A, Mandras N, Roana J, et al. Probiotics and gut health in infants: A preliminary case - control observational study about early treatment with Lactobacillus reuteri DSM 17938. Clin Chim Acta (2015) 451:82-7. doi: 10.1016/j.cca.2015.02.027

24. Savino F, Cordisco L, Tarasco V, Locatelli E, Di Gioia D, Oggero R, et al. Antagonistic effect of Lactobacillus strains against gas-producing coliforms isolated from colicky infants. BMC Microbiol. (2011) 11:157. doi: 10.1186/1471-2180-11-157

25. Di Gioia D, Aloisio I, Mazzola G. Bifidobacteria : their impact on gut microbiota composition and their applications as probiotics in infants. Appl Microbiol Biotechnol. (2014) 98:563-77. doi: 10.1007/s00253-013-5405-9

26. Aloisio I, Santini C, Biavati B, Dinelli G, Cenci A, Chingwaru W, et al. Characterization of Bifidobacterium spp. strains for the treatment of enteric disorders in newborns. Appl Microbiol Biotechnol. (2012) 96:1561-76. doi: $10.1007 / \mathrm{s} 00253-012-4138-5$

27. Li Y, Shimizu T, Hosaka A, Kaneko N, Ohtsuka Y, Yamashiro Y. Effects of Bifidobacterium breve supplementation on intestinal flora of low birth weight infants. Pediatrics Int. (2004) 46: 509-15. doi: 10.1111/j.1442-200x.2004.01953.x

28. Wada M, Nagata S, Saito M, Shimizu T, Yamashiro Y, Matsuki T, et al. Effects of the enteral administration of Bifidobacterium breve on patients undergoing chemotherapy for pediatric malignancies. Support Care Cancer (2010) 18:751-9. doi: 10.1007/s00520-009-0711-6

29. Costeloe K, hardy P, Juszczak E, Wilks M, Millar MR. Bifidobacterium breve BBG-001 in very preterm infants: a randomised controlled phase 3 trial. Lancet (2016) 387:649-60. doi: 10.1016/s0140-6736(15)01027-2

30. Patole SK, Rao SC, Keil AD, Nathan EA, Doerthy DA, Simmer KN. Benefits of Bifidobacterium breve M-16V supplementation in preterm neonates - a retrospective cohort study. PLoS ONE (2016) 11:e0150775. doi: 10.1371/journal.pone. 0150775

31. Mogna L, Del Piano M, Mogna G. Capability of the two microorganisms Bifidobacterium breve $\mathrm{B} 632$ and Bifidobacterium breve BR03 to colonize the intestinal microbiota of children. J Clin Gastroenterol. (2014) 48:S37-9. doi: 10.1097/MCG.0000000000000234

32. Simone M, Gozzoli C, Quartieri A, Mazzola G, Di Gioia D, Amaretti A, et al. The Probiotic Bifidobacterium breve B632 Inhibited the growth of Enterobacteriaceae within colicky infant microbiota cultures. Biomed Res Int. (2014) 2014:1-7. doi: 10.1155/2014/301053

33. Quagliariello A, Aloisio I, Bozzi Cionci N, Luiselli D, Auria GD, Martinezpriego L, et al. Effect of Bifidobacterium breve on the intestinal microbiota of coeliac children on a gluten free diet : a pilot study. Nutrients (2016) 8:660-76. doi: $10.3390 /$ nu 8100660

34. Malinen E, Kassinen A, Rinttila T, Palva A. Comparison of real-time PCR with SYBR Green I or 59 -nuclease assays and dot-blot hybridization with rDNAtargeted oligonucleotide probes in quantification of selected faecal bacteria. Microbiology (2003) 149:269-77. doi: 10.1099/mic.0.25975-0

35. Penders J, Vink C, Driessen C, London N, Thijs C, Stobberingh EE. Quantification of Bifidobacterium spp., Escherichia coli and Clostridium difficile in faecal samples of breast-fed and formula-fed infants by real-time PCR. FEMS Microbiol Lett. (2005) 243:141-7. doi: 10.1016/j.femsle.2004.11.052

36. Rinttila T, Kassinen A, Malinen E, Krogius L, Palva A. Development of an extensive set of $16 \mathrm{~S}$ rDNA-targeted primers for quantification of pathogenic and indigenous bacteria in faecal samples by real-time PCR. J Appl Microbiol. (2004) 97:1166-77. doi: 10.1111/j.1365-2672.2004. 02409.x

37. Castillo M, Martín-Orúe SM, Manzanilla EG, Badiola I, Martín M, Gasa J. Quantification of total bacteria, enterobacteria and lactobacilli populations in pig digesta by real-time PCR. Vet Microbiol. (2006) 114:165-70. doi: $10.1016 /$ j.vetmic.2005.11.055 
38. Penders J, Thijs C, Vink C, Stelma FF, Snijders B, Kummeling I, van den Brandt PA, Stobberingh EE. Factors influencing the composition of the intestinal microbiota in early infancy. Pediatrics (2006) 118:511-21. doi: 10.1542/peds.2005-2824

39. Haarman M, Knol J. Quantitative real-time PCR assays to identify and quantify fecal Bifidobacterium species in infants receiving a prebiotic infant formula. Appl Environ Microbiol. (2005) 71:2318-24. doi: 10.1128/AEM.71.5.2318-2324.2005

40. Leser TD, Amenuvor JZ, Jensen TK, Lindecrona RH, Boye M, Møller K. Culture-independent analysis of gut bacteria : the pig gastrointestinal tract microbiota revisited. Appl Environ Microbiol. (2002) 68:673-90. doi: 10.1128/AEM.68.2.673

41. Barr RG, Kramer MS, Boisjoly C, McVey-White L, Pless IB. parental diary of infant cry and fuss behaviour. Arch Dis Childh. (1988) 63:380-7. doi: 10.1136/adc.63.4.380

42. Lane MM, Czyzewski DI, Mph BPC, Shulman RJ. Reliability and validity of a modified bristol stool form scale for children. J Pediatr. (2011) 159:437-41.e1. doi: 10.1016/j.jpeds.2011.03.002

43. Aloisio I, Mazzola G, Corvaglia LT, Tonti G, Faldella G, Biavati B, et al. Influence of intrapartum antibiotic prophylaxis against group B Streptococcus on the early newborn gut composition and evaluation of the anti-Streptococcus activity of Bifidobacterium strains. Appl Microbiol Biotechnol. (2014) 98:6051-60. doi: 10.1007/s00253-0145712-9

44. Altschul SF, Gish W, Miller W, Myers EW, Lipman DJ. Basic local alignment search tool. J Mol Biol. (1990) 215:403-10. doi: 10.1016/S0022-2836(05)80360-2

45. Lee ZMP, Bussema C, Schmidt TM. rrn DB: Documenting the number of rRNA and tRNA genes in bacteria and archaea. Nucleic Acids Res. (2009) 37:489-93. doi: 10.1093/nar/gkn689

46. Indrio F, Martini S, Francavilla R, Corvaglia L, Cristofori F, Martini S. Epigenetic matters: the link between early nutrition, microbiome, and long-term health development. Front Pediatr. (2017) 5:178. doi: 10.3389/fped.2017.00178

47. Liepke C, Adermann K, Raida M, Magert H-J, Forssmann W, Zucht HD. Human milk provides peptides highly stimulating the growth of bifidobacteria. Eur J Biochem. (2002) 269:712-8. doi: 0.1046/j.00142956.2001.02712.x

48. Obermajer T, Grabnar I, Benedik E, Tušar T, Pikel TR, Mis NF, Matijašić BB, Rogelj I. microbes in infant gut development: placing abundance within enviromental, clinical and growth parametres. Sci Rep. (2017) 7:11230. doi: 10.1038/s41598-017-10244-x

49. Stuebe A. The risks of not breastfeeding. Rev Obstet Gynecol. (2009) 2:222-31. doi: 10.3909/riog0093

50. Favier CF, Vaughan EE, De Vos WM, Akkermans ADL. Molecular monitoring of succession of bacterial communities in human neonates. Society (2002) 68:219-26. doi: 10.1128/AEM.68.1.219

51. McFadden A, Gavine A, Renfrew M, Wade A, Buchanan P, Taylor J, et al. Support for healthy breastfeeding mothers with healthy term babies. Cochrane Database Syst Rev. (2017) 2:1-224. doi: 10.1002/14651858.CD0 01141
52. García Marqués S, Chillón Martínez R, González Zapata S, Rebollo Salas M, Jiménez Rejano JJ. Tools assessment and diagnosis to infant colic: a systematic review. Child Care Health Dev. (2017) 43:481-8. doi: 10.1111/cch. 12454

53. Xu M, Wang J, Wang N, Sun F, Wang L, Liu X. The efficacy and safety of the probiotic bacterium Lactobacillus reuteri DSM 17938 for infantile colic : a meta-analysis of randomized controlled trials. PLoS ONE (2015) 10:1-16. doi: 10.1371/journal.pone.0141445

54. Magne F, Puchi Silva A, Caravajal B, Gotteland M. The elevated rate of cesarean section and its contribution to non-communicable chronic diseases in latin america : the growing involvement of the microbiota. Front Pediatr. (2017) 5:192. doi: 10.3389/fped.2017. 00192

55. Kuhle S, Tong OS, Woolcott CG. Pediatric obesity and pregnancy association between caesarean section and childhood obesity : a systematic review and meta-analysis. Obes Rev. (2015) 16:295-303. doi: 10.1111/obr. 12267

56. De Moura EG, Lisboa PC, Passos MCF. Neonatal programming of neuroimmunomodulation - role of adipocytokines and neuropeptides. Neuroimmunomodulation (2008) 15:176-88. doi: 10.1159/0001 53422

57. Mulligan CM, Friedman JE. Maternal modifiers of the infant gut microbiota : metabolic consequences. J Endocrinol. (2017) 235:R1-12. doi: 10.1530/JOE-17-0303

58. Baldassarre ME, Di Mauro A, Mastromarino P, Fanelli M, Martinelli D, Urbano F, et al. Administration of a multi-strain probiotic product to women in the perinatal period differentially affects the breast milk cytokine profile and may have beneficial effects on neonatal gastrointestinal functional symptoms. a randomized clinical trial. Nutrients (2016) 8:1-13. doi: 10.3390/nu81 10677

59. Härtel C, Pagel J, Spiegler J, Buma J, Henneke P, Viemann D, et al. Lactobacillus acidophilus/Bifidobacterium infantis probiotics are associated with increased growth of VLBWI among those exposed to antibiotics. Sci Rep. (2017) 7:1-11. doi: 10.1038/s41598-01706161-8

Conflict of Interest Statement: Authors LM and MP were employed by the company Biolab Research Srl, Novara, Italy, performing all Research \& Development activities for Probiotical SpA, Novara, Italy.

The other authors declare that the research was conducted in the absence of any commercial or financial relationships that could be construed as a potential conflict of interest.

Copyright (C) 2018 Aloisio, Prodam, Giglione, Bozzi Cionci, Solito, Bellone, Baffoni, Mogna, Pane, Bona and Di Gioia. This is an open-access article distributed under the terms of the Creative Commons Attribution License (CC BY). The use, distribution or reproduction in other forums is permitted, provided the original author(s) and the copyright owner are credited and that the original publication in this journal is cited, in accordance with accepted academic practice. No use, distribution or reproduction is permitted which does not comply with these terms. 